\title{
A Pilot Clinical Study of Ocular Prosthesis Fabricated by Three- dimensional Printing and Sublimation Technique
}

\author{
Bo Ram Kim ${ }^{1}$, So Hyun Kim ${ }^{1,2}$, JaeSang Ko, Seung Woon Baek ${ }^{1}$, Yung Kyung Park ${ }^{3}$, Yoon Jung Kim ${ }^{4}$, Jin Sook Yoon ${ }^{1}$ \\ ${ }^{1}$ Department of Ophthalmology, Institute of Vision Research, Severance Hospital, Yonsei University College of Medicine, Seoul, \\ Korea \\ ${ }^{2}$ Department of Biomedical Engineering, Yonsei University College of Medicine, Seoul, Korea \\ ${ }^{3}$ Department of Design, Ewha Womans University, Seoul, Korea \\ ${ }^{4}$ Ewha Color Design Research Institute, Ewha Womans University, Seoul, Korea
}

\begin{abstract}
Purpose: We sought to evaluate the safety and effectiveness of patient-specific ocular prostheses produced by three-dimensional (3D) printing and the sublimation technique. A comparison with prostheses produced using manual manufacturing methods was then performed.

Methods: To confirm the biological and physiochemical safety, cytotoxicity, systemic acute toxicity, intradermal reaction, and skin sensitization tests were conducted according to the International Organization for Standardization guidelines. The compressive strength of the prostheses was also tested. Further, a case series of three patients who wore the 3D printed prostheses for more than eight hours daily for 4 weeks was executed. Self-assessments by these individuals using a questionnaire and safety evaluations focusing on the occurrence of conjunctival inflammation or allergic reactions according to the Cornea and Contact Lens Research Unit criteria by slit-lamp examination and similarity assessment were completed.

Results: The 3D printed ocular prostheses met the necessary qualifications per the biological and physiochemical safety tests, showing the absence of cytotoxicity, acute systemic toxicity, intradermal reactivity, and skin-sensitizing potency. Also, there was no difference in strength test results between previous ocular prostheses and the 3D printed ones. Self-assessment by the patients yielded satisfactory results, with no significant difference in the level of satisfaction reported for the 3D printed and previous handmade ocular prostheses. The 3D printed prosthesis did not trigger any side effects in the conjunctival sac and showed similar objective findings with respect to the color of the iris, sclera, and vessel patterns.

Conclusions: Our study confirms the biologic and physiochemical safety of 3D-printed ocular prostheses created using computer-aided design technology and a sublimation technique. The patients' questionnaires and the judgment of the ophthalmologists/ocularists showed that the 3D printed ocular prosthesis was acceptable in function and appearance through a case series report.
\end{abstract}

Key Words: Anophthalmos, Artificial eye, Computer-aided design, Sublimation technique, Three-dimensional printing

Received: October 12, 2020 Final revision: November 9, 2020 Accepted: November 12, 2020

Corresponding Author: Jin Sook Yoon, MD, PhD. Department of Ophthalmology, Institute of Vision Research, Severance Hospital, Yonsei University College of Medicine, 50-1 Yonsei-ro, Seodaemun-gu, Seoul 03722, Korea. Tel: 82-2-2228-3570, Fax: 82-2-312-0541, E-mail: yoonjs@ yuhs.ac
Ocular prostheses are important rehabilitation tools for anophthalmic patients, improving their quality of life by restoring the symmetrical appearance of the face [1-3]. However, current production methods still fail to make the patient's eye look natural while simultaneously ensuring that the area feels comfortable. Ocular prostheses can be 
produced by ready-made stock shells or can be handmade $[4,5]$. Of these, handmade ocular prostheses garner higher results in satisfaction surveys because they are custom-made to fit the patient [6]. However, the success of the handmade technique is not only extremely dependent on the experience of the ocularist but is also time-consuming, labor-intensive, and costly to adopt [4].

Recently, the application of three-dimensional (3D) printing technology in the medical field is gaining attention [7]. 3D printing has been introduced in the medical field as a means to develop teaching aids for students, preplan surgical guides for surgeons, and therapeutic tools for patients $[7,8]$. Most 3D printers produce objects by laminating materials based on 3D model data by way of an additive process [9]. With the development of 3D printing technology and the increase in the variety of materials that can be used for printing, it has become possible to create objects that cannot be easily manufactured by conventional methods. Alam et al. [10] was among the first investigators to use 3D printing to design ocular prostheses in 2017. The team conducted a study on the eyes of two patients using computer-aided design (CAD) technology that produced more comfortable prostheses in less time. However, 3D printing technology was limited at that time to only being able to print the scleral form; the iris and vessels still required painting by hand. Until recently, no objective and standardized technique of manufacturing ocular prostheses using 3D printers has existed. Our research team recently reported on a novel semi-automated method using 3D printing technology and a sublimation transfer printing technique for manufacturing customized ocular prostheses [11]. In our previous study, we used a 3D printer to produce ocular prostheses. After printing the ocular prosthesis shape, the iris and blood vessels were printed onto the ocular prosthesis using a dye sublimation transfer technique.

The purpose of the present study is to evaluate the safety and effectiveness of patient-specific ocular prosthesis creation by 3D printer in a clinical case series of three patients. Through this study, we confirmed that 3D printing paired with the mentioned sublimation transfer technique could be substituted for traditional handmade manufacturing methods of ocular prostheses.

\section{Materials and Methods}

\section{Ethics statement}

The study was approved by the institutional review board of the College of Medicine, Yonsei University (12019-0064). Informed consent was obtained from the included patients and the study strictly adhered to the tenets of the Declaration of Helsinki.

\section{Participants}

This was a single-center, preliminary case series report. The major inclusion criteria included the following: age between 19 and 75 years, status post-enucleation or -evisceration, and duration of ocular prosthesis use for at least one year before enrollment. The exclusion criteria included the following: age younger than 19 years, signs of conjunctival and socket contracture or inflammation, and exposure or infection of the orbital implant.

\section{D data acquisition and 3D printing of the ocular pros- thesis}

The process of semi-automated 3D printing of ocular prostheses was performed as previously described [11]. After obtaining 3D modeling data, the scleral model was printed using DS131 (Carima, Seoul, Korea), a digital light-processing (DLP) 3D printer with biocompatible photopolymer resin (FotoTec DLP.A; Dreve, Unna, Germany). Unlike in the previous paper [11], where, during the process of applying color and shape to the scleral surface, the photo of the other normal eye was previously taken and transferred, in this study, a digital image of the iris and blood vessels of the patient's other eye was generated using a color diagnostic tool and transferred onto the scleral surface using a sublimation transcription technique (Fig. 1A1F). Subsequent contents are the same as those existing in our previous paper [11]. Lastly, the ocular prosthesis was completed by coating the surface with transparent polymethyl methacrylate. This technology makes it possible to produce personalized ocular prostheses objectively and consistently and has the advantage of reducing the time and skills required to produce ocular prostheses. 

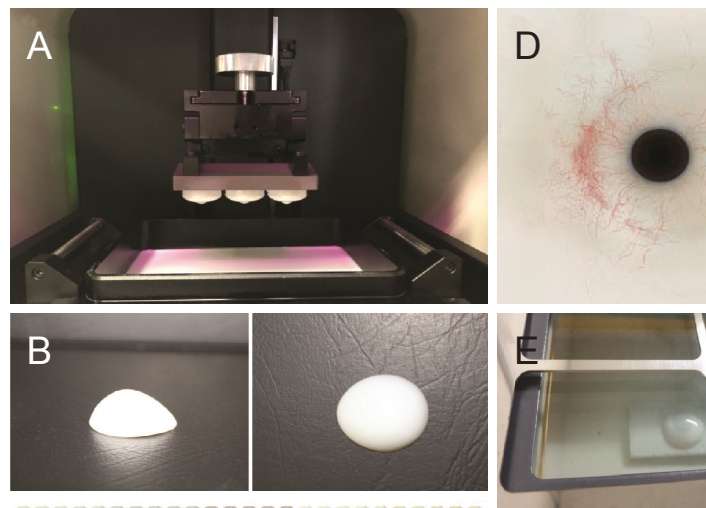

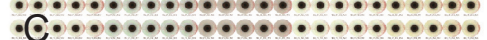

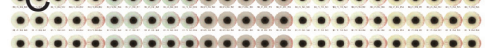
$\bullet \bullet \bullet \bullet \bullet \bullet \bullet \bullet \bullet \bullet \bullet \bullet \bullet \bullet \bullet \bullet \bullet \bullet \bullet \bullet$ $\bullet \bullet \bullet \bullet \bullet \bullet \bullet \bullet \bullet \bullet \bullet \bullet \bullet \bullet \bullet \bullet \bullet \bullet \bullet \bullet \bullet \bullet \bullet \bullet$ $\bullet \bullet \bullet \bullet \bullet \bullet \bullet \bullet \bullet \bullet \bullet \bullet \bullet \bullet \bullet \bullet \bullet \bullet \bullet \bullet \bullet$ $\bullet \bullet \bullet \bullet \bullet \bullet \bullet \bullet \bullet \bullet \bullet \bullet \bullet \bullet \bullet \bullet \bullet \bullet \bullet \bullet$

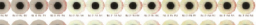
$+$ $F$

KOREAN STANDARD OF IRIS OF ARTIFIIIAL EVE

Fig. 1. Process of sublimation transfer printing for three-dimensional (3D) ocular prosthesis creation. (A) The ocular prosthesis manufacturer selects a scleral 3D modeling file with customized details of the patient's conjunctiva and then manufactures the sclera product using a digital light-processing 3D printer. (B) After washing the impurities remaining on the surface of the sclera product with alcohol, the sclera product is completed. (C) The graphic data that most closely matches the image of the patient's healthy eyes from standardized color sets are selected to match the iris color and pattern of the patient. (D) After printing the graphic data on a transfer paper with a sublimation transfer inkjet printer, the transfer paper is dried at room temperature. (E) The sclera product is placed in the center of the sublimation transfer machine table, the center of the transfer paper is aligned to the marked pupil center of the sclera, and sublimation transfer printing is initiated. (F) The final product of the 3D ocular prosthesis with sublimation transfer technique.

\section{Color diagnostic tool}

We created a standard image dataset for enabling images to be sublimated on the sclera surface conveniently and quickly, even if not overseen by an expert ocularist. Standard image data were created to match the patient's normal eye. The representative image of $4,000 \times 4,000$ pixels (1,500 pixels/inch) consisted of iris colors, iris patterns, scleral colors, vessel colors, and vessel densities based on the standardized dataset. The process of compiling the dataset of the seven iris colors and six iris patterns was performed as previously reported [12]. Then, we further developed a color diagnostics tool to apply the colors of the standardized dataset objectively and consistently; nine iris colors, six iris patterns, nine scleral colors, seven vessel

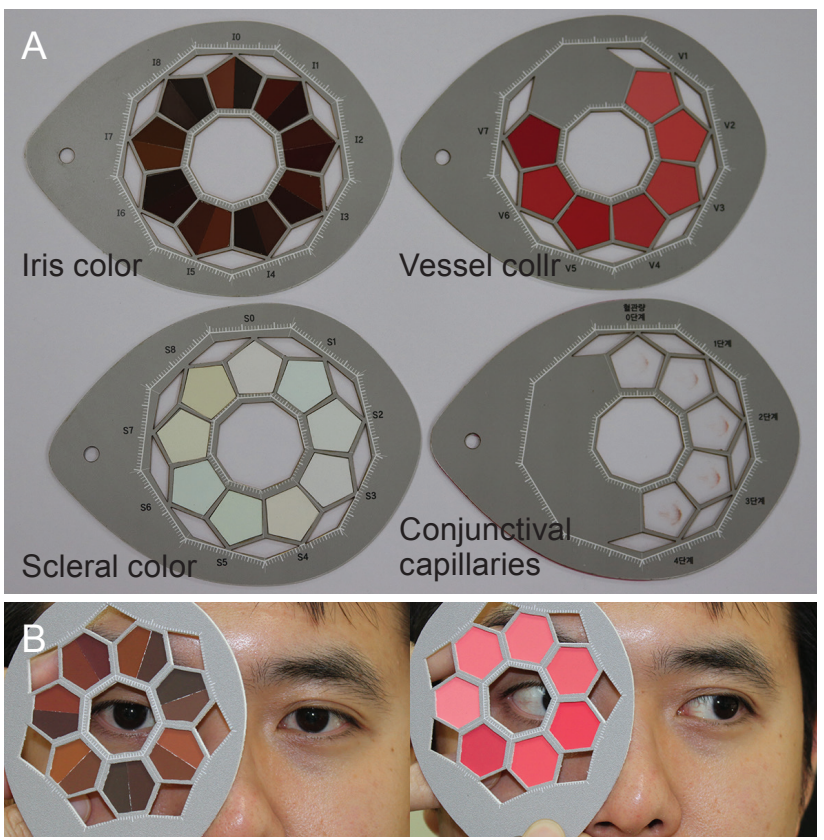

Fig. 2. Color diagnostic tool for sublimation. (A) The $12 \times 9$-cmsized oval-shaped tool was printed on a paper with a $2.5-\mathrm{cm}$ opening at the center to compare iris color, vessel color, scleral color and vessel densities. (B) The photographs below show the process for matching iris color and vessel color. Informed consent for publication of the clinical images was obtained from the patient.

colors and five vessel densities (Fig. 2A, 2B). The $12 \times$ 9-cm-sized oval-shaped tool was printed on a paper with a $2.5-\mathrm{cm}$ opening at the center. Each page was composed of the iris colors, iris patterns, scleral colors, vessel colors, and vessel densities aligned in a radiating formation surrounding the center. Then, we placed an image of the normal eye in the middle of the color diagnostic tool and selected the standard color or shape that most closely resembles it. Finally, the image data were directly printed on the scleral surface using the sublimation transfer technique.

\section{Evaluation of the biological and physicochemical safety of the 3D ocular prosthesis}

Safety was assessed using cytotoxicity, systemic acute toxicity, intradermal reaction, and skin sensitization tests according to the International Organization for Standardization 10993 Series Biological Evaluation of Medical Devices guidelines [13]. Ten 3D-printed ocular prostheses were used for each test. Compressive strength testing using a universal test machine (model 5569; Instron, Norwood, 
MA, USA) was conducted at a constant crosshead speed $(19.8-25.6 \mathrm{~mm} / \mathrm{min})$.

\section{Clinical case series of three patients}

We conducted a clinical observational study involving three eyes of three patients who were assessed after wearing 3D printed ocular prostheses according to three categories: self-assessment by questionnaire performed by the patient, conjunctival socket evaluation by slit-lamp imaging performed by an ophthalmologist, and assessments of ocular similarity performed by an ocularist.

On the day of the first visit, a semi-automated 3D printed ocular prosthesis was manufactured. The patients were asked to wear the ocular prosthesis for more than eight hours a day for 4 weeks. 3D printed ocular prosthesis management was conducted in a manner similar to that of the patients' existing prostheses.

\section{1) Self-assessment by questionnaire}

We evaluated the patients' satisfaction in cosmetic and functional aspects by questionnaire (Supplemental Table 1). The patients were asked to fill out the study questionnaire at 2 and 4 weeks. Patients were asked to give answers to questions on topics including the color and shape (cosmetic evaluation) and experiences with symptoms including the levels of eye crust (frequency, color, volume, and viscosity), pruritus, pain, dryness, and eyelid swelling (functional evaluation).

\section{2) Safety assessment by slit-lamp imaging}

A slit-lamp examination with photography was performed to grade conjunctival changes (Supplemental Table 2). Conjunctival inflammation and allergic reactions in both the lower and upper palpebral areas were evaluated using a grading scale (grades $0-4$ ) according to Cornea and Contact Lens Research Unit standards [14].

\section{3) Similarity assessment}

An assessment of the similarity between the 3D printed ocular prosthesis and the normal eye accroding to iris color, scleral color, iris pattern, and vessel pattern was performed by an expert ocularist (SWB) as very similar, similar, roughly similar, different, or very different.

\section{Results}

\section{Evaluation of the safety and strength of the 3D ocular prosthesis}

The 3D printed ocular prosthesis passed the biocompatibility standards for cytotoxicity, acute systemic toxicity, intradermal toxicity, and sensitization. Cell cytotoxicity in vitro testing using mouse-derived fibroblasts (L-929 cells) revealed no isolation of intracytoplasmic granules, cell lysis, or the inhibition of cell growth. No case of animal death was observed due to acute systemic toxicity during in vivo testing using ICR mice and no general symptoms, weight changes, or specific autopsy findings related to the administration of the test substance were observed. In a Hartley guinea pig maximization in vivo test, no general symptoms, weight changes, or death were observed and the material from which the prothesis was made was shown to be a non-sensitizing substance of Magnusson and Kligman grade 0 [15]. Further, an animal intracutaneous (intradermal) reactivity in vivo test was conducted using New Zealand White rabbits and no abnormal symptoms or weight changes related to the administration of the test substance were observed and the 3D printed ocular prosthesis was considered to trigger no intradermal reaction (Table 1).

The previous handmade ocular prostheses $(\mathrm{n}=10)$ had a mean compressive strength of $2,540 \pm 381 \mathrm{~N}$, while the 3D-printed ocular prostheses $(n=10)$ showed a value of

Table 1. Safety tests for the three-dimensional printed ocular prostheses

\begin{tabular}{lll}
\hline Test & Standard & \multicolumn{1}{c}{ Result } \\
\hline Cytotoxicity & ISO 10993-5 & No cytotoxicity \\
Acute systemic toxicity & ISO 10993-11 & No significant systemic toxicity \\
Intradermal toxicity & ISO 10993-10 & No significant toxicity or irritation \\
Sensitization & ISO 10993-10 & No dermal sensitization \\
\hline
\end{tabular}


$2,588 \pm 407 \mathrm{~N}$. There were no significant differences $(p>$ 0.05 ) between the two types of ocular prosthesis.

\section{Clinical case series of three patients}

Three patients (one female aged 26 years and two males aged 33 and 35 years) were enrolled in this study. All patients wore their $3 \mathrm{D}$ printed ocular prostheses daily for a period of 4 weeks.

\section{1) Self-assessment by questionnaire}

Fig. 3A and 3B presents representative photos and slitlamp details of the three enrolled patients. Self-assessments performed by patients using the questionnaire were conducted to evaluate cosmetic and functional outcomes of the 3D printed ocular prostheses. After wearing their prostheses for 4 weeks, the patients were "very satisfied" with both the color and shape as part of the cosmetic evaluation. Separately, the frequency, color, volume, and viscosity of eye crust were assessed and, in all four categories, the survey results indicated improvements were observed after wearing the $3 \mathrm{D}$ printed ocular prostheses.

A
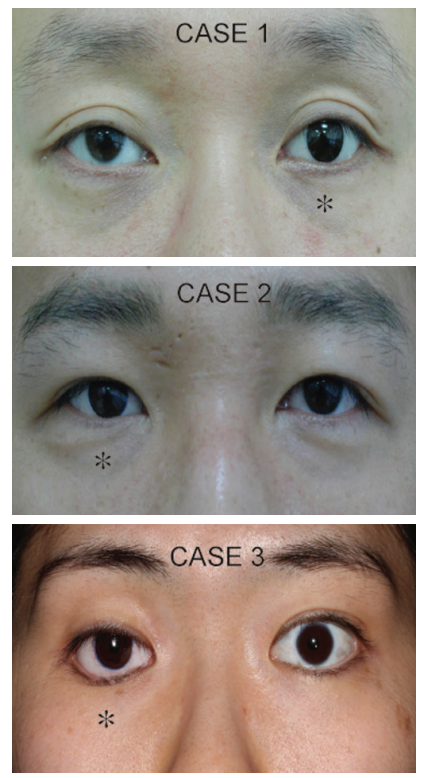

B
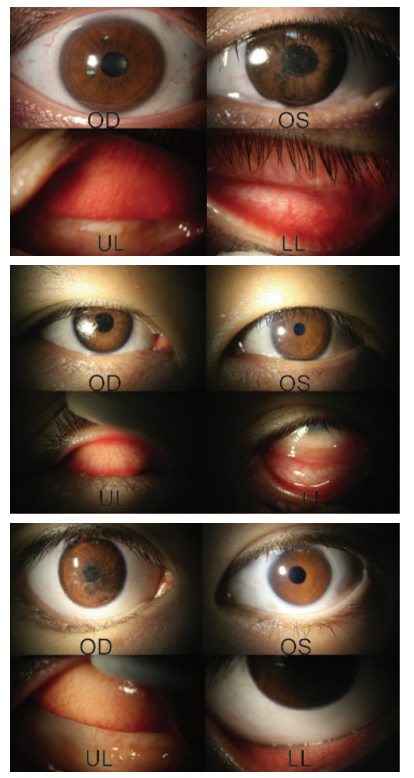

Fig. 3. Photographs of three patients' normal eyes and anophthalmic eyes. (A) Gross photos of the three patients at primary gaze. (B) Slit-lamp photos of the normal eye, the ocular prosthesis and the upper and lower conjunctiva of the anophthalmic socket. The ocular prosthesis is marked by an asterisk. $\mathrm{OD}=$ right eye; $\mathrm{OS}$ $=$ left eye; $\mathrm{UL}=$ upper lid; $\mathrm{LL}=$ lower lid. Informed consent for publication of the clinical images was obtained from the patients.
Pruritis was not experienced by any patient after wearing the 3D printed ocular prostheses. Also, no newly developed pain was noted in two patients; moreover, previously reported existing pain disappeared after wearing the 3D printed ocular prosthesis in the third patient. The level of ocular dryness did not change in two patients and improved in the third patient. After wearing the 3D printed ocular prostheses for 4 weeks, eyelid swelling was not observed in any of the patients. During the final evaluation conducted 4 weeks later, patients reported improvements in both cosmetic and functional satisfaction levels compared to their existing ocular prostheses (Supplemental Table 3).

\section{2) Safety assessment by slit-lamp imaging}

During the safety assessment, conjunctival inflammation and allergic reactions were graded in accordance with the Cornea and Contact Lens Research Unit criteria by an ophthalmologist. The conjunctival inflammation did not change in two patients and showed an improvement from grade 2 to 1 after wearing the 3D printed ocular prosthesis in the third patient. No instance of conjunctival allergic reaction after 4 weeks was observed in any of the patients (Supplemental Table 2).

\section{3) Similarity assessment}

All three prostheses were deemed by the expert ocularist to show "very similar" results with regard to iris color, scleral color, iris pattern, and vessel patterns when compared with the patients' other (real) eyes.

\section{Discussion}

The world's first ocular prosthesis is believed to have been made by the Egyptians in that prostheses constructed from stone, silver, and gold were found in mummies and statues $[1,16,17]$. The usage of glass as a prosthetic material gradually resulted in the use of dental acrylic and methyl methacrylate [1]. More recently, current CAD 3D printing technology has been adopted to create ocular prostheses $[10,18]$. In our previous paper, we published the details of a novel semi-automated method that incorporated 3D printing technology and a sublimation transfer printing technique to manufacture customized ocular prostheses [11]. Moreover, we applied a new technology — a color diagnos- 
tic tool-in this paper. Here, during the process of making the ocular prosthesis, an ocularist drew the iris after observing the patient's normal eye with acrylic ink and used red silk fibers to imitate the vessels traditionally [19]. This stage plays an important role in satisfying the patient and ensures the color and pattern of the ocular prosthesis will be delivered appearing most similar to those of the healthy eye. In our previous paper, we sublimated the photograph of the other normal eye; however, when the photograph is sublimated on the surface of the prosthesis, the printed image is often different from the actual ocular image, which is a limitation. So, we created graphic data by applying a color diagnostic tool, which is a simple and fast technique, reduces errors in the imaging color process, and has the advantage of requiring minimal artistic skill. There are several reports covering $3 \mathrm{D}$ printing of ocular prostheses $[10,18,20]$. To the best of our knowledge, however, this is the first paper to evaluate the safety and efficacy of such in a prospective, exploratory clinical case series.

Still, papers on the development of ocular prostheses using 3D printing technology have explored a number of aspects. Various authors of the published papers commonly performed 3D volume reconstruction using computed tomography, designed the data using CAD, and printed $3 \mathrm{D}$ ocular prostheses to overcome the drawbacks of the previous ocular prostheses. Ye et al. [20] printed using standard polymer filament and melted polylactic acid and the patient was reported to be satisfied with the appearance of their prosthesis, but the paper lacks details on the exact follow-up period or other complications. Another report used CAD to develop a design that was hollow on the inside to reduce the weight of the prosthesis and printed with biocompatible polymethyl methacrylate. The satisfaction survey result indicated satisfaction was apparent with both the shape and comfort level of the ocular prosthesis [10]. Lastly, Ruiters et al. [18] reported the case of a 68-year-old male who, after 6 months of wearing a 3D-printed ocular prosthesis, reported no significant discomfort. However, this was just a single case report and was limited because additional efforts to find an agent that would adhere to the ophthalmic socket were needed.

The difference between these existing published studies and our research is that, for the first time in our paper, a sublimation transfer technique with a color diagnostic tool was used to omit the process of direct painting on the $3 \mathrm{D}$ printed material. Second, to confirm that it was biological- ly safe and to test whether the intensity was similar to that of the previous prosthesis, experiments certified by the International Organization for Standardization (cytotoxicity, acute systemic toxicity, intradermal toxicity, sensitization tests) were conducted. Third, a case series was conducted that included three patients who performed a self-assessment, received a conjunctival socket assessment performed by an ophthalmologist, and a similarity assessment conducted by an ocularist. This series showed that there was no shortcoming evident as compared with customized manufactured products. So far, the studies focused on creating ocular prostheses with 3D printing technology have not focused on safety and effectiveness. Among the aforementioned papers, the studies briefly asked about patient satisfaction with appearance or discomfort during wearing, but there have been no systematic papers published on safety and effectiveness to date.

When creating an agenda using the 3D printing technology introduced in this paper, it is expected to save time compared to creating a conventional handmade customized ocular prosthesis. In addition, the 3D printing approach can save existing patient data and reuse it afterward, reducing the costs of production and making it easier to replace lost or produce multiple prostheses. Relatively inexperienced ocularists are able to create prostheses easily using a semi-automatic process. In this report, we were able to clinically verify the validity of these advantages that were discussed in our previous report [11].

In conclusion, we introduce the details of a new attempt to evaluate the safety and effectiveness of 3D printed ocular prostheses created using CAD and a sublimation technique. Although the number of patients was small and the duration of wearing the ocular prostheses was relatively short, the results are encouraging. Similar studies involving larger sample sizes that explore our results in more detail will help to validate the benefits of this advanced technology when compared with traditional methods. Also, long-term research is needed for the next 3 to 5 years when considering the replacement period of the eye. In the near future, we predict the wide-spread use of 3D printed prostheses as part of the growth of personalized medicine will occur. 


\section{Conflict of Interest}

No potential conflict of interest relevant to this article was reported.

\section{Acknowledgements}

This work was supported by the Bio \& Medical Technology Development Program through the National Research Foundation (NRF) funded by the Korean government, MSIP (2015M3A9E2067031) and by the Basic Science Program through the NRF funded by the Ministry of Science and ICT (2016M3A9E9941746).

\section{Supplemental Material}

Supplemental Table 1-3 are available from: https://doi. org $/ 10.3341 /$ kjo.2020.0125.

\section{References}

1. Raizada K, Rani D. Ocular prosthesis. Cont Lens Anterior Eye 2007;30:152-62.

2. Chin K, Margolin CB, Finger PT. Early ocular prosthesis insertion improves quality of life after enucleation. Optometry 2006;77:71-5.

3. Goiato MC, dos Santos DM, Bannwart LC, et al. Psychosocial impact on anophthalmic patients wearing ocular prosthesis. Int J Oral Maxillofac Surg 2013;42:113-9.

4. Cain JR. Custom ocular prosthetics. J Prosthet Dent 1982;48:690-4.

5. Ow RK, Amrith S. Ocular prosthetics: use of a tissue conditioner material to modify a stock ocular prosthesis. $J$ Prosthet Dent 1997;78:218-22.

6. Gunaseelaraj R, Karthikeyan S, Kumar MN, et al. Custom-made ocular prosthesis. J Pharm Bioallied Sci 2012;4(Suppl 2):S177-9.

7. Chae MP, Rozen WM, McMenamin PG, et al. Emerging applications of bedside 3D printing in plastic surgery. Front Surg 2015;2:25.

8. Mardis NJ. Emerging technology and applications of 3D printing in the medical field. Mo Med 2018;115:368-73.

9. Ngo TD, Kashani A, Imbalzano G, et al. Additive manufacturing (3D printing): a review of materials, methods, applications and challenges. Compos B Eng 2018;143:172-96.

10. Alam MS, Sugavaneswaran M, Arumaikkannu G, Mukherjee B. An innovative method of ocular prosthesis fabrication by bio-CAD and rapid 3-D printing technology: a pilot study. Orbit 2017;36:223-7.

11. Ko J, Kim SH, Baek SW, et al. Semi-automated fabrication of customized ocular prosthesis with three-dimensional printing and sublimation transfer printing technology. Sci Rep 2019;9:2968.

12. Jang H, Yoon J, Kim YJ, Park Y. Classification of iris colors and patterns in Koreans. Healthc Inform Res 2018;24:22735.

13. Northup SJ. Safety evaluation of medical devices: US Food and Drug Administration and International Standards Organization guidelines. Int J Toxicol 1999;18:275-83.

14. Terry RL, Schnider CM, Holden BA, et al. CCLRU standards for success of daily and extended wear contact lenses. Optom Vis Sci 1993;70:234-43.

15. Magnusson B, Kligman AM. The identification of contact allergens by animal assay. The guinea pig maximization test. J Invest Dermatol 1969;52:268-76.

16. Kelley JJ. History of ocular prostheses. Int Ophthalmol Clin 1970;10:713-9.

17. McCord CP. Artificial eyes: the early history of ocular prostheses. J Occup Med 1965;7:61-8.

18. Ruiters S, Sun Y, de Jong S, et al. Computer-aided design and three-dimensional printing in the manufacturing of an ocular prosthesis. Br J Ophthalmol 2016;100:879-81.

19. Fernandes AU, Goiato MC, Batista MA, Santos DM. Color alteration of the paint used for iris painting in ocular prostheses. Braz Oral Res 2009;23:386-92.

20. Ye X, Wang S, Zhu Y, et al. Automatic design and fabrication of a custom ocular prosthesis using 3D volume difference reconstruction (VDR). IEEE Access 2018:6;14339-46. 
Supplementary Table 1. The self-assessment questionnaire

\begin{tabular}{|c|c|c|c|c|c|c|c|c|}
\hline Category & & & Visit & & & Grade & & \\
\hline \multirow[t]{3}{*}{ Cosmetic } & & & & Very unsatisfied & Unsatisfied & Neutral & Satisfied & Very satisfied \\
\hline & Color & & $4 \mathrm{wk}$ & & & & & \\
\hline & Shape & & $4 \mathrm{wk}$ & & & & & \\
\hline \multirow[t]{28}{*}{ Function } & Eye crust & & & None & Biweekly & Weekly & Daily & Constantly \\
\hline & & Frequency & Initial & & & & & \\
\hline & & & $2 \mathrm{wk}$ & & & & & \\
\hline & & & $4 \mathrm{wk}$ & & & & & \\
\hline & & & & Clear & White & Cream & Yellow & \\
\hline & & Color & Initial & & & & & \\
\hline & & & $2 \mathrm{wk}$ & & & & & \\
\hline & & & $4 \mathrm{wk}$ & & & & & \\
\hline & & & & None & Small & Moderate & Large & \\
\hline & & Volume & Initial & & & & & \\
\hline & & & $2 \mathrm{wk}$ & & & & & \\
\hline & & & $4 \mathrm{wk}$ & & & & & \\
\hline & & & & None & Low & Moderate & High & \\
\hline & & Viscosity & Initial & & & & & \\
\hline & & & $2 \mathrm{wk}$ & & & & & \\
\hline & & & $4 \mathrm{wk}$ & & & & & \\
\hline & & & & None & Mild & Moderate & Severe & Very severe \\
\hline & Pruritis & & Initial & & & & & \\
\hline & & & $2 \mathrm{wk}$ & & & & & \\
\hline & & & $4 \mathrm{wk}$ & & & & & \\
\hline & Pain & & Initial & & & & & \\
\hline & & & $2 \mathrm{wk}$ & & & & & \\
\hline & & & $4 \mathrm{wk}$ & & & & & \\
\hline & Dryness & & Initial & & & & & \\
\hline & & & $2 \mathrm{wk}$ & & & & & \\
\hline & & & $4 \mathrm{wk}$ & & & & & \\
\hline & Eyelid swelling & & & Present & None & & & \\
\hline & & & $4 \mathrm{wk}$ & & & & & \\
\hline \multirow[t]{3}{*}{ Final evaluation } & & & & Very unsatisfied & Unsatisfied & Neutral & Satisfied & Very satisfied \\
\hline & $\begin{array}{l}\text { Cosmetic } \\
\text { satisfaction }\end{array}$ & & $4 \mathrm{wk}$ & & & & & \\
\hline & $\begin{array}{l}\text { Functional } \\
\text { satisfaction }\end{array}$ & & $4 \mathrm{wk}$ & & & & & \\
\hline
\end{tabular}


Supplemental Table 2. Safety assessment results according to slit-lamp imaging

\begin{tabular}{lcccc}
\hline Category & Visit & Patient 1 & Patient 2 & Patient 3 \\
\hline Conjunctival inflammation & Initial & Grade 1 & Grade 2 & Grade 1 \\
& $4 \mathrm{wk}$ & Grade 1 & Grade 1 & Grade 1 \\
Allergic reaction & Initial & Grade 1 & Grade 1 & Grade 1 \\
& $4 \mathrm{wk}$ & Grade 1 & Grade 1 & Grade 1 \\
\hline
\end{tabular}

Supplemental Table 3. Self-assessment questionnaire results reported by the patients

\begin{tabular}{|c|c|c|c|c|c|c|}
\hline Category & & & Visit & Patient 1 & Patient 2 & Patient 3 \\
\hline \multirow[t]{2}{*}{ Cosmetic } & Color & & $4 \mathrm{wk}$ & Very satisfied & Very satisfied & Very satisfied \\
\hline & Shape & & $4 \mathrm{wk}$ & Very satisfied & Very satisfied & Very satisfied \\
\hline \multirow[t]{22}{*}{ Function } & Eye crust & Frequency & Initial & None & Constantly & Daily \\
\hline & & & $2 \mathrm{wk}$ & None & Daily & Daily \\
\hline & & & $4 \mathrm{wk}$ & None & Daily & Daily \\
\hline & & Color & Initial & Clear & White & Yellow \\
\hline & & & $2 \mathrm{wk}$ & Clear & White & Yellow \\
\hline & & & $4 \mathrm{wk}$ & Clear & White & Yellow \\
\hline & & Volume & Initial & None & Small & Large \\
\hline & & & $2 \mathrm{wk}$ & None & Small & Large \\
\hline & & & $4 \mathrm{wk}$ & None & Small & Moderate \\
\hline & & Viscosity & Initial & None & Moderate & Moderate \\
\hline & & & $2 \mathrm{wk}$ & None & Low & Moderate \\
\hline & & & $4 \mathrm{wk}$ & None & Low & Moderate \\
\hline & Pruritis & & Initial & None & None & None \\
\hline & & & $2 \mathrm{wk}$ & None & None & None \\
\hline & & & $4 \mathrm{wk}$ & None & None & None \\
\hline & Pain & & Initial & None & Mild & None \\
\hline & & & $2 \mathrm{wk}$ & None & Mild & None \\
\hline & & & $4 \mathrm{wk}$ & None & None & None \\
\hline & Dryness & & Initial & None & Severe & Severe \\
\hline & & & $2 \mathrm{wk}$ & None & Mild & Severe \\
\hline & & & $4 \mathrm{wk}$ & None & Mild & Severe \\
\hline & Eyelid swelling & & $4 \mathrm{wk}$ & None & None & None \\
\hline \multirow[t]{2}{*}{ Final evaluation } & Cosmetic satisfaction & & $4 \mathrm{wk}$ & Very satisfied & Very satisfied & Very satisfied \\
\hline & Functional satisfaction & & $4 \mathrm{wk}$ & Very satisfied & Very satisfied & Very satisfied \\
\hline
\end{tabular}

